\title{
A study on disease burden and treatment among patients attending teleconsultation during lockdown period
}

\author{
Mounika Yedlapalli', Sai Pasupula Kiran ${ }^{2 *}$, Sravani Potti ${ }^{1}$, \\ Devikala A. ${ }^{1}$, Chandrakala Kambar ${ }^{1}$
}

${ }^{1}$ Department of Pharmacology, ${ }^{2}$ Department of Psychiatry, Guntur Medical College, Guntur, Andhra Pradesh, India

Received: 15 February 2021

Revised: 15 March 2021

Accepted: 17 March 2021

*Correspondence:

Dr. Sai Pasupula Kiran,

Email: nottymedico@gmail.com

Copyright: (C) the author(s), publisher and licensee Medip Academy. This is an open-access article distributed under the terms of the Creative Commons Attribution Non-Commercial License, which permits unrestricted non-commercial use, distribution, and reproduction in any medium, provided the original work is properly cited.

\begin{abstract}
Background: Covid-19 has been announced as a pandemic by the World Health Organization. To maintain social distancing effectively, the Government of India announced a complete lockdown on March 25th, 2020. As there are no proper transport facilities for patients who need health care services, the Government of Andhra Pradesh started 104 sevakendram as a helpline. Medical services were also provided by this 104 sevakendram through teleconsultation with doctors. The aim and objectives of the study were to study the disease pattern and treatment given to patients attending teleconsultations, to analyze the disease burden among the patients attending teleconsultation, to study the treatment given to the patients attending teleconsultation.

Methods: It is a retrospective, observational, and analytical study. After prior IEC clearance and permission from teleconsultation authority, we did the study at Dr.YSR Aarogyasri health care trust, Guntur. Data regarding all calls connected to 104 was collected. Detailed information of the calls related to the Medical officer about the age, gender, disease pattern, and treatment given to the patient was collected. Statistical analysis was done using MS excel software. Results: On average, 104 sevakendram received two hundred valid teleconsultations per day. Most of the calls are related to anxiety and these calls constitute twenty four percent. Treatment given was according to probable diagnosis, mostly symptomatic and continuation of the same treatment.

Conclusions: 104 sevakendram has played a vital role in meeting the medical and health requirements of the people suffering from different diseases during the lockdown period.
\end{abstract}

Keywords: Lockdown, Teleconsultation, Disease pattern

\section{INTRODUCTION}

COVID -19 is the Pandemic caused by the virus severe acute respiratory syndrome coronavirus, originated in China. ${ }^{1-3}$ The transmission with high infectivity of virus made India's government issue lockdown on March 25 to manage the Covid 19 outbreak. As a result, there are no modes of transport with most hospitals closed, and people became panic to come out from home, which made the innovative technology of teleconsultation. ${ }^{4}$ So the government of Andhra Pradesh announced 104 sevakendram as a helpline, which filled the gap between people, physicians, and health systems without increasing the spread of SARS CoV-2.

"Tele" is a Greek word meaning "distance, "and "mederi" is a Latin word meaning "to heal." 5 Teleconsultation played a crucial role in disaster management history and reputed organizations like NASA and NATO also used these services. ${ }^{6-9}$ 
Aim

To study the disease pattern and treatment given to patients attending teleconsultations.

\section{Objectives}

To analyze the disease burden among the patients attending teleconsultation. To study the treatment given to the patients attending teleconsultation.

\section{METHODS}

\section{Study type}

Retrospective, observational, and analytical study

\section{Study population and center}

All the patients who attended teleconsultation through 104 sevakendram during the lockdown period are the study population. The study was conducted at Dr. YSR Aarogyasri health care trust, Guntur.

\section{Study duration}

The study duration is two months that is from April 2020 to May 2020.

After prior IEC clearance and permission from teleconsultation authority, the study was done at Dr. YSR Arogyasri health care trust, Guntur.

Data regarding all calls connected to 104 was collected. Detailed information of the calls related to the Medical officer about the age, gender, disease pattern, and prescription given to the patient was collected. We used MS excel software for statistical analysis.

\section{RESULTS}

On average, 104 sevakendram received two thousand two hundred valid teleconsultations per day (Figure 1).

Executives used to attend the calls first. Of these around, 671(31\%) calls were about COVID-19 disease, i.e., about spreading of virus, symptoms, preventive methods, testing centers, and hospital facilities.

Specially trained executives answered these calls. These calls were gradually decreased after two weeks. Of all these valid calls, around 200 calls (9\%) per day were connected to Medical officers.

\section{Demographic details}

The mean age of the patients was 38.5 years. Thirty-nine percent of the total population are males, and females constitute sixty-one percentage. Percentage of complaints in each system was plotted (Figure 2).

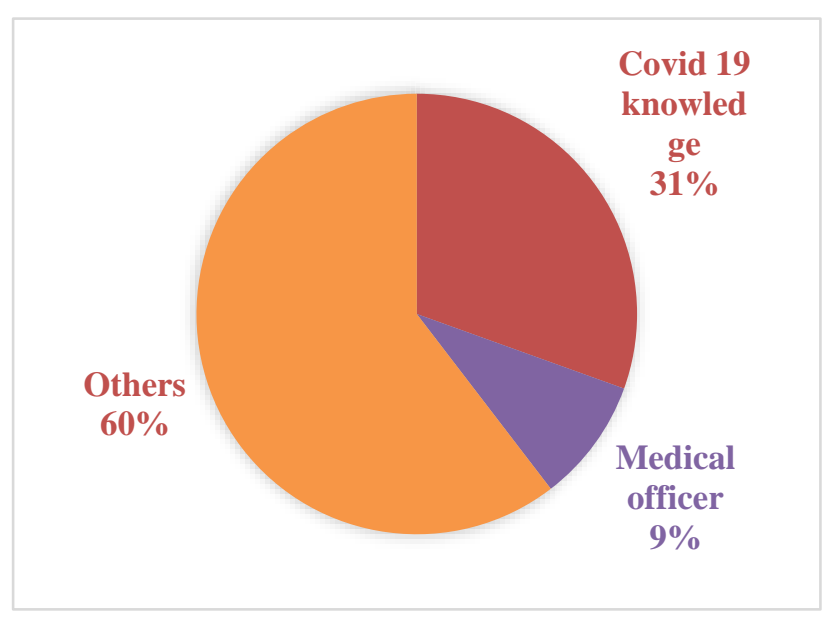

Figure 1: Calls connected to 104.

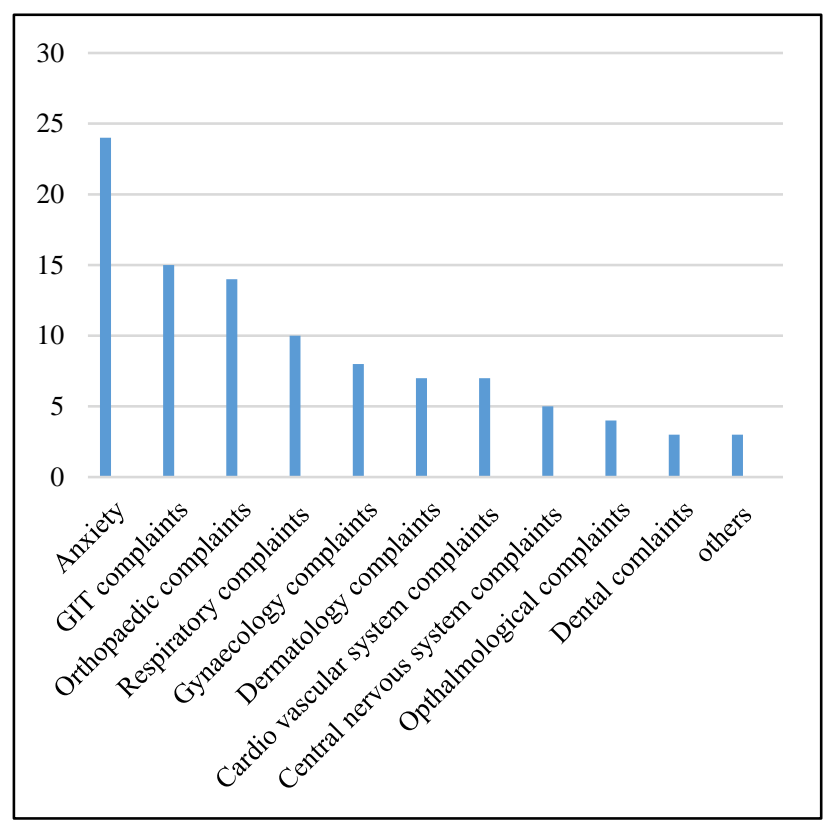

Figure 2: Percentage of complaints in each system.

\section{Anxiety related calls}

During the 1st two weeks, $45 \%$ of the calls connected to medical officers were about generalized anxiety. Some of these patients are counselled by medical officers, and some patients who need intense counselling were connected to a counsellor. And some patients who need medication were prescribed low dose Benzodiazepines or $Z$ drugs for a short period after confirmation with the Psychiatrist and were asked to follow up after ten days (Figure 3).

After two weeks, the percentage of calls about COVIDrelated anxiety was decreased to $24 \%$, and it is around $8 \%$ during the next month. 


\section{Gastrointestinal tract (GIT) conditions}

The percentage of calls complaining about symptoms related to GIT is $15 \%$ (Figure 4).

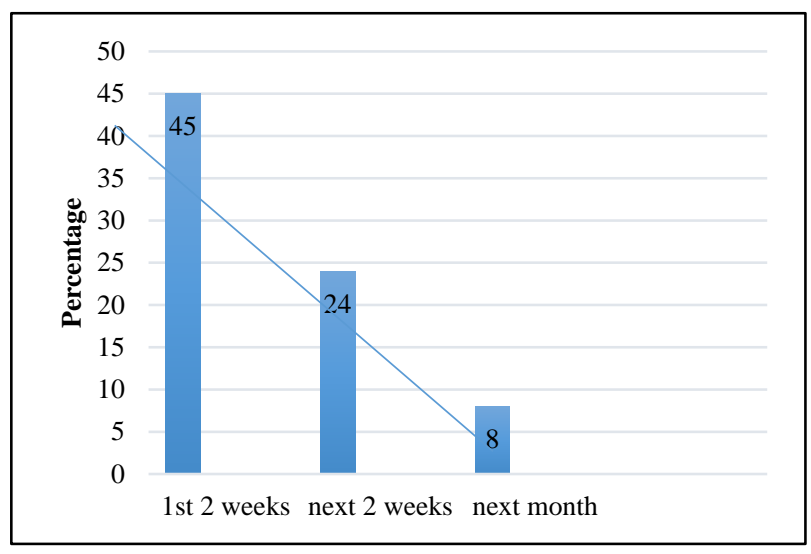

Figure 3: Anxiety related calls.

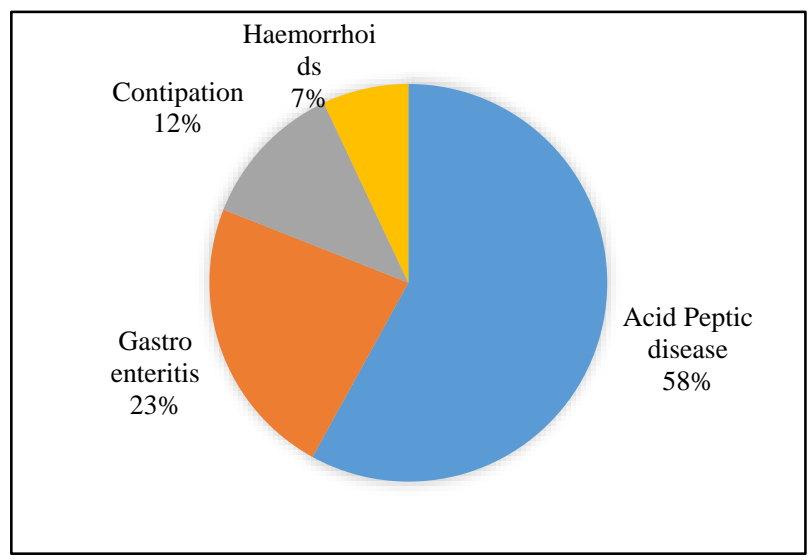

Figure 4: GIT conditions.

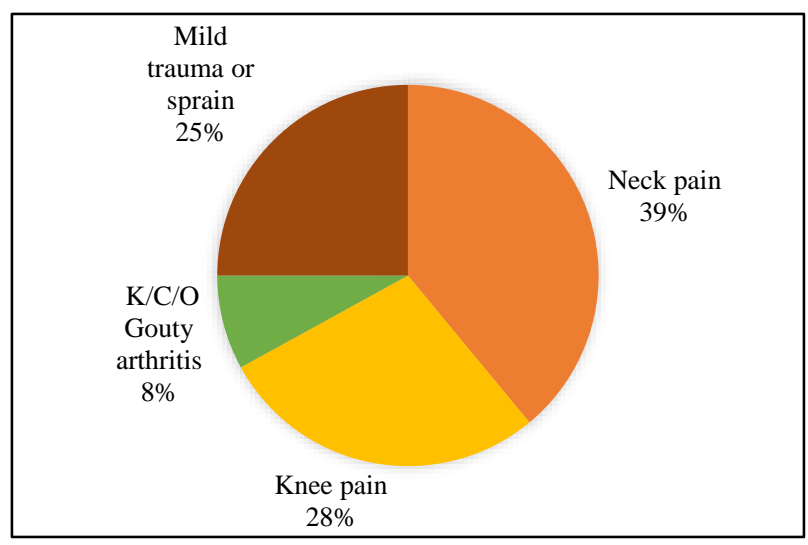

Figure 5: Orthopaedic conditions.

Acid peptic disease (58\%): Proton pump inhibitors were given and advised about regular food habits.

Gastroenteritis with vomitings or diarrhea $(23 \%)$ : symptomatic treatment with 5-HT3 antagonist or prokinetics, lactobacillus powder mixed with water, and increased fluid intake was advised. Some patients who have more episodes of vomitings or diarrhea with pain abdomen or generalized symptoms like fever or severe weakness due to the fluid loss were given antibiotics like Fluoroquinolones, oral rehydration salts, and antipyretics if required.

Constipation (12\%): Laxatives, fiber-rich diet was advised.

Hemorrhoids (7\%): Laxatives, fiber-rich diet, local muscle relaxant ointment was advised.

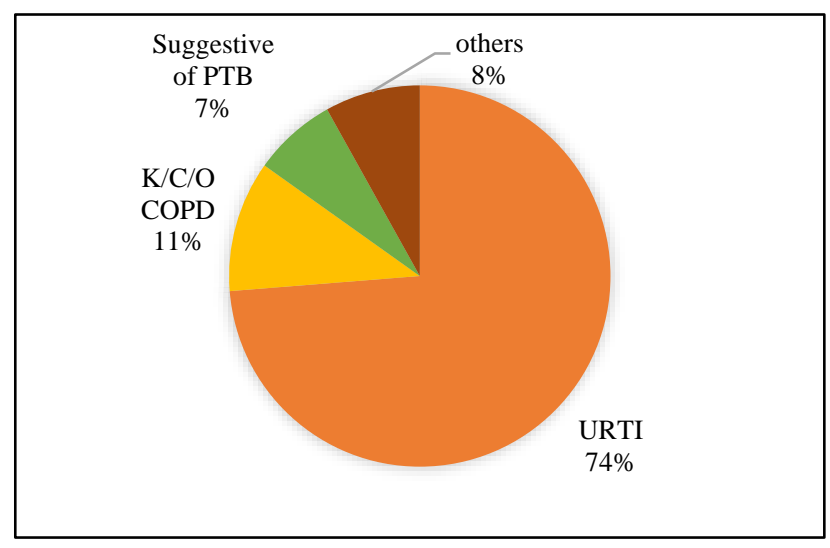

Figure 6: Respiratory system conditions.

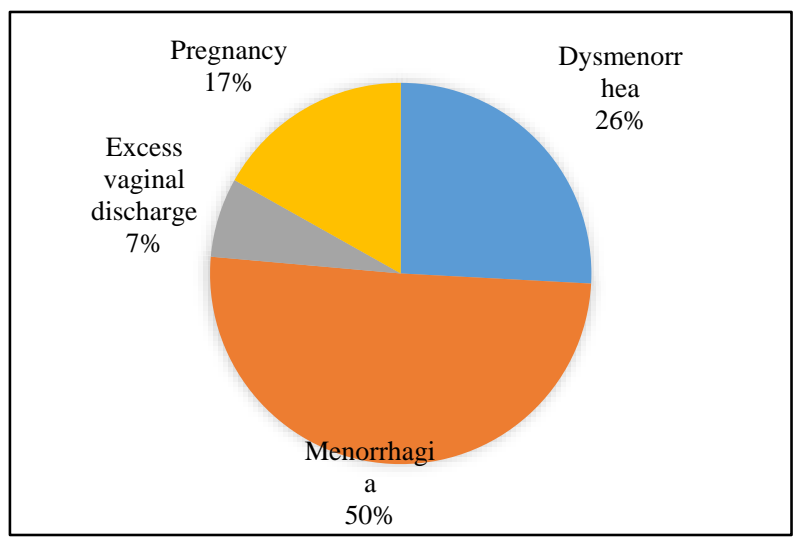

Figure 7: Gynaecology and obstetrics conditions.

\section{Orthopaedic conditions}

These constitute $14 \%$ of the calls connected to the medical officer (Figure 5).

Neck pain, neck pain radiating to hand (39\%): Analgesics, muscle relaxants, and postural modifications were advised.

Knee pain (28\%): Analgesics for symptomatic relief for a short duration and behavioral modification was advised.

Known case of Gouty arthritis (8\%): Analgesics excluding aspirin, diet modification, methylprednisolone, depending on the severity, were prescribed. 
Mild trauma or sprain (25\%): Analgesics, Crepe bandage if required, was advised.

\section{Respiratory system conditions}

These constitute $10 \%$ of the calls connected to the medical officer (Figure 6).

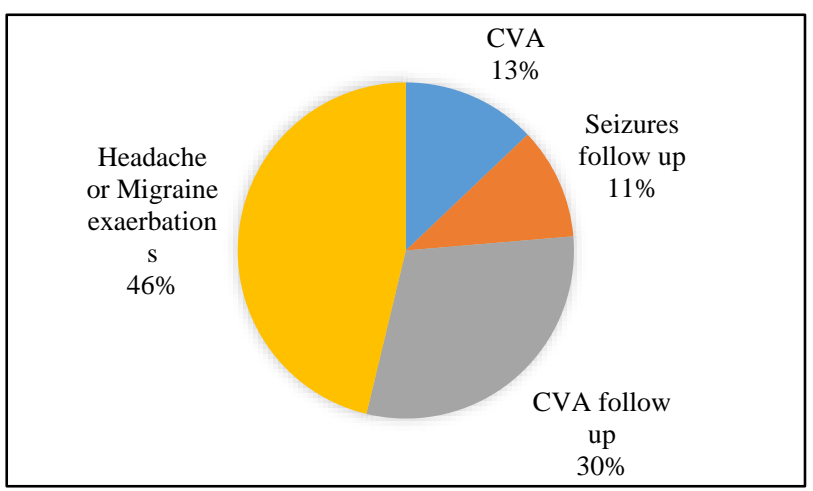

Figure 8: Central nervous system conditions.

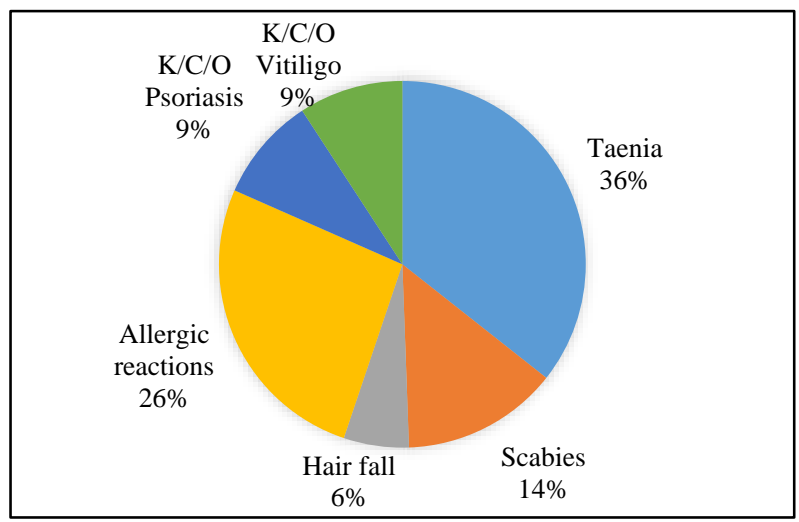

Figure 9: Dermatological conditions.

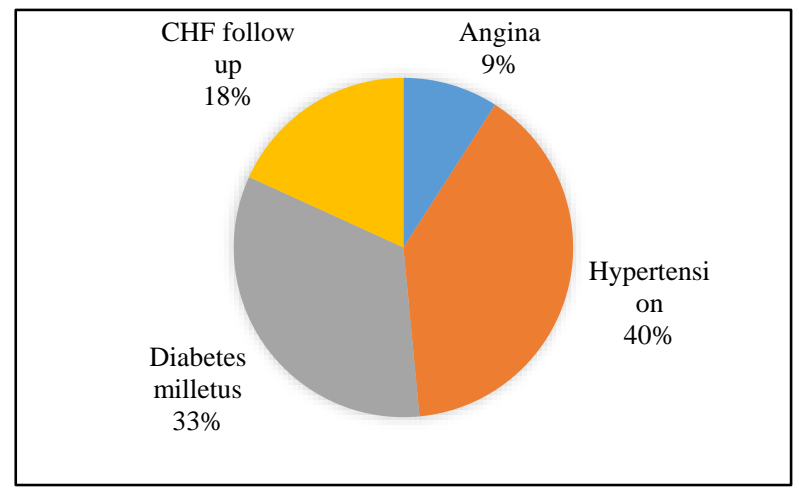

Figure 10: Cardiovascular system conditions.

Upper respiratory tract infection (URTI) (73\%): symptomatic treatment with antihistamines for the common cold, mucolytic agents for the cough with expectoration, warm saline water gargling, and analgesics for pharyngitis were given after taking good travel history and covid 19 contact history. Those who are suspected of the covid disease are informed to get tested for RTPCR of SARS CoV-2, and his/her details were sent to primary health care service providers of his/their area for monitoring.

Known case of COPD (11\%): continue same treatment; bronchodilators were given if required.

Suggestive of pulmonary tuberculosis (PTB) (7\%): Advised for confirmation with acid-fast staining and sputum sample culture in the nearby primary health center.

\section{Gynaecology and obstetric conditions}

These constitute $8 \%$ of the calls connected to the medical officer (Figure 7).

Dysmenorrhoea (23\%): Analgesics for symptomatic relief and reassurance was given.

Menorrhagia (45\%): for those with no apparent cause and menstrual-related excess bleeding- excess fluid intake, iron supplementation, and reassurance was given. For those with suspected pathology- iron supplementation, antifibrinolytics if required, were prescribed and advised to follow up with a gynecologist if symptoms persist.

Excess vaginal discharge with itching (6\%): antifungal drugs for both partners and antibiotics were prescribed (tetracyclines and metronidazole) if necessary.

Pregnancy (15\%): Folic acid, iron, and calcium supplementation, tetanus injection if available, general precautions were advised.

\section{Central nervous system conditions}

These constitute $5 \%$ of the calls connected to the medical officer (Figure 8).

Cerebro vascular accidents (CVA) (12\%): General protective measures were advised, and the call was forwarded to 108 for immediate admission to hospital.

Known case of seizures (10\%): General protective measures were advised, and the call was forwarded to 108 for immediate admission to hospital.

Follow-ups of CVA (28\%): advised to continue same treatment, physiotherapy, and symptomatic treatment was given if required.

Headache or migraine exacerbation (43\%): analgesic with antiemetic depending on symptoms was advised.

\section{Dermatological conditions}

These constitute $7 \%$ of the calls connected to the medical officer (Figure 9). 
Taenia (31\%): Topical antifungal ointment, general precautions were advised.

Scabies (12\%): Permethrin ointment for all the family members and general precautions were advised.

Allergic reactions (23\%): Avoidance of allergen and antihistamines were given.

Chickenpox (8\%): Analgesics, symptomatic treatment, antibiotic for prophylaxis of secondary infections, and general precautions were advised.

Follow up of psoriasis (8\%): Advised to continue the same treatment with steroid.

Follow up of vitiligo (9\%): Advised to continue same treatment.

Hairfall (5\%): Vitamin and Iron supplementation were advised.

\section{Cardiovascular system}

These constitute $7 \%$ of the calls connected to the medical officer (Figure 10).

Angina (12\%): Glyceryl trinitrate sublingual tablet, general precautions were advised, and the call was connected to 108 for immediate admission to hospital.

Known case of chronic heart failure (CHF) (23\%): continue same treatment, general precautions, diuretics added if required.

Hypertension (39\%): Antihypertensives as per age and comorbidities, lifestyle modification

Known case of diabetes mellitus with or without heart failure (26\%): Most of the patients advised continuing the same treatment. Only for a few patients treatment was changed as per blood sugar levels and other complaints.

\section{Opthalmology conditions}

These constitute $4 \%$ of the calls connected to the medical officer.

Conjunctivitis/foreign body sensation: topical antihistamine drops and antibiotic drops, if required, are prescribed.

\section{Dental conditions}

These constitute $3 \%$ of the calls connected to the medical officer.

Toothache: analgesics were advised.
Dental caries: analgesics and antibiotics were prescribed if required.

Stomatitis: vitamin B complex, betadine mouth wash, topical analgesic+ anesthetic ointment was prescribed if required.

\section{Miscellaneous calls}

Some of the calls are related to Hydroxychloroquine's availability for the known case of rheumatoid arthritis or systemic lupus erythmatosis or poly arthralgia. They are taking Hydroxychloroquine for few months. Some calls were regarding the availability of chemotherapeutic drugs like capecitabine- colorectal carcinoma, Gefitinib: nonsmall cell lung carcinoma, Mesalazine for Inflammatory bowel disease, Leuprolide for benign prostatic hyperplasia, and psychotropic drugs, especially antidepressants, mood stabilizers.

\section{Immunization schedules}

Some calls were received about the delay in immunization schedules from parents. Reassurance was given that there will be no harm even it was delayed by 2 weeks.

\section{DISCUSSION}

The current research advances the earlier literature in studying the disease patterns and treatment among the patients attending teleconsultation during the lockdown period. COVID-19 affects the entire health system through its direct effect as a contagious disease and its ability to alter the overall mortality and burden of illness through the impact on non-communicable conditions. Collection of accurate and comprehensive global data regarding COVID-19 and other significant causes of disease burden should be a public health priority. 10 Telemedicine services have become one of the best solutions for this, and they played a crucial role in serving the needy during the Pandemic of COVID illness.

\section{Generalized anxiety disorders}

Coronaphobia is an excessive triggered response of fear of contracting the virus causing COVID-19, leading to extreme concern over physiological symptoms, significant stress about personal and occupational loss, whose treatment includes increased reassurance and safetyseeking behaviors, and general avoidance of places and situations, causing marked impairment in daily life functioning. ${ }^{11}$

Earlier literature on pandemic found that major pandemic or epidemic outbreaks are anticipated to impact individuals and society negatively. ${ }^{12}$ The current study also found that majority of the calls in the initial phase of the pandemic were constituted by anxiety-related COVID illness. 
Sharing COVID symptoms with typical flu symptoms and lockdown with concepts of isolation and quarantine made the individuals more anxious.

According to the study of Islam et al. conducted in Bangladesh, the findings suggest that high levels of panic and generalized anxiety in Bangladesh were observed. Specialized mental healthcare services for the Bangladeshi people during this COVID-19 outbreak could potentially reduce panic and anxiety. ${ }^{13}$

According to the study of Shi et al conducted in China, the prevalence of symptoms of depression, anxiety, insomnia, and acute stress was high in this sample of study participants from China during the COVID-19 Pandemic, especially among patients with confirmed or suspected COVID-19 and their family members and friends, people with occupational exposure risks, and residents of Hubei province. $^{14}$

Pervasive intervention measures, including quarantine and delays in returning to work, were closely associated with adverse mental health outcomes. Individuals with personality disorder and a history of psychiatric disorders are more prone to anxiety-related disorders. ${ }^{15}$

\section{Acid peptic disease}

Previous research on the causes of peptic ulcer disease stated that stress with minimal physical activity would contribute to acid peptic disorders. ${ }^{16,17}$ In the current study, we found that fifty-eight percent of the calls under GIT are related to Acid peptic disorders for which proton pump inhibitors were prescribed. Lack of daily income due to lock down and closure of gyms, playgrounds made people stay at home, which decreased the physical activity. These factors ultimately lead to acid peptic disorders.

\section{Neck pain}

Among the orthopedic conditions, more number of calls are related to neck pain. Work from home resulted in a lack of proper working setup- improper working position, and also increased working hours may attribute to cause neck pain. Work-related neck disorders are common problems in office workers, especially among those who are intensive computer users.18,19

The discomfort postures due to the low viewing angle of the screen and the position of the chair and table may shorten the soft tissues and cause muscle tension, weakness, and fatigue. ${ }^{20}$

\section{Headache}

Stress due to covid, increased screen time may cause headache, and we received many calls relating to symptoms of migraine. The study of Al-Hashel et al about migraine shows that their research provides "real-world" data regarding the negative impact of the COVID-19
Pandemic on migraine. The majority of respondents struggled with an increase in migraine frequency and severity. Around $10 \%$ of patients transformed into chronic migraine during lockdown. ${ }^{21}$

High levels of screen time exposure are associated with migraine in young adults. ${ }^{22}$

During the 2003 severe acute respiratory distress syndrome epidemic, healthcare workers mandatorily wore the protective N95 face-mask. Lee, Einar Wilder-Smith administered a survey to healthcare workers to determine risk factors associated with the development of headaches.37.3\%respondents reported face-maskassociated headaches. ${ }^{23}$

\section{Respiratory conditions}

Calls regarding the respiratory conditions are more on upper respiratory tract symptoms, which constituted seventy-three percentage of the needs related to respiratory disorders.

\section{Gynecological conditions}

Most of the calls are related to menstrual problems like menorrhagia and dysmenorrhea. Stress will cause menstrual irregularities, and high anxiety with low physical activity made individuals vulnerable to stress-causing menstrual discomfort.

\section{Other conditions}

The migraine type, hypertension, foreign body sensation in the eye, and toothache are the standard calls received under neurology, cardiology, ophthalmology, and dental specialties.

There are miscellaneous calls regarding Hydroxychloroquine's availability: With regards to COVID-19, chloroquine (CQ) and hydroxychloroquine (HCQ) are identified as possible therapeutic drugs potentially useful in combating COVID-19.24 HydroxyChloroquine was brought under the scheduled $\mathrm{H} 1$ drugs of the Drugs and Cosmetic Rules, 1945, after it has been recommended as prophylaxis for healthcare providers and household contacts of COVID-19 confirmed cases by the Indian Council of Medical Research. Following the order, the State drugs control department has ordered all chemists and druggists associations not to give the drug to anyone without a doctor's prescription. Patients already taking HCQ for Rheumatoid arthritis or SLE or Poly arthralgia were not given HCQ even though they have the old medicine. So the patients called up to 104 sevakendram for HCQ. 104 sevakendram has made arrangements to provide the new prescription mentioning the indication for HCQ by the local primary health care doctor.

Some calls were received about the availability of antidepressants, mood stabilizers, and chemo therapeutic 
drugs. The local primary health center doctor was contacted to know the local pharmacies where these drugs will be available. With the help of a pharmacist or ASHA worker, these drugs were supplied to patients.

\section{Limitations}

As it is a teleconsultation, patient may not be able to tell about all the symptoms, but attempts were made to collect as much information from the patient by spending more time with each patient. Many patients didn't call for followup. As there is no video consultation, physical examination, and diagnostic tests, mostly probable diagnosis was considered, and treatment was given according to that. To overcome this limitation government has started a new program by the name "Telemedicine," where is a chance for video consultation.

\section{CONCLUSION}

104 sevakendram through teleconsultation approach has played a vital role in meeting the medical and health requirements of the people suffering from different diseases during the lockdown period. This study shows the increased incidence of anxiety-related disorders during the initial phase of lockdown, which needs to be addressed, and this is well managed by the 104 sevakendram. Still, there is a need to increase awareness about teleconsultation among the general population, reducing the gap between patients and health care workers.

\section{ACKNOWLEDGEMENTS}

We would like to thanks Dr. YSR Aarogyasri Health Care Trust.

\section{Funding: No funding sources}

Conflict of interest: None declared

Ethical approval: The study was approved by the Institutional Ethics Committee, Guntur Medical College, Guntur

\section{REFERENCES}

1. World Health Organization (WHO), Coronavirus, 2020. https://www.who.int/healthtopics/coronavirus\#tab=tab. Accessed on 10 January, 2021.

2. Mackenzie JS, Smith DW. COVID-19: a novel zoonotic disease caused by a coronavirus from China: what we know and what we don't know. Journal of microbiology, Australia. 2020;MA20013.

3. WHO Novel coronavirus (2019-nCoV) situation reports.

https://www.who.int/emergencies/diseases/novelcoro navirus-2019/situation-reports. Accessed on $10^{\text {th }}$ January, 2021.

4. Monaghesh E, Hajizadesh A. The role of telehealth during COVID-19 outbreak: a systematic review based on current evidence. BMC Public Health. 200;20.

5. Dasgupta A, Deb S. Telemedicine: A New Horizon in Public Health in India. Indian Journal of Telemedicine. 2008;33(1).

6. World health organization, Telemedicine: opportunities and developments in member states Global Observatory for eHealth series. https://www.who.int/goe/publications/goe_telemedici ne_2010.pdf. Accessed on $10^{\text {th }}$ January, 2021

7. Bashshur R. Technology serves the people: The story of a cooperative telemedicine project by NASA, the Indian Health Service and the Papago people, Superintendent of Documents, Washington DC: US Government Printing Office. 1980.

8. Doarn CR, Latifi R, Poropatich RK, Sokolovich N, Kosiak D, Hostiuc F et al. Development and Validation of Telemedicine for Disaster Response The North Atlantic Treaty Organization Multinational System. Telemedicine journal and e-health. 2018;24(9):657-668.

9. Wicklund, E. Harvey's aftermath brings mHealth, telehealth to the forefront. 2017. https://mhealthintelligence.com/news/harveysaftermath-brings-mhealth-telehealth-to-the-forefront. Last accessed on $10^{\text {th }}$ January, 2021.

10. Azarpazhoob MR, Moroyatar N, Avan A, Divani A, Yassi N et al. COVID-19 Pandemic and burden of non communicable diseases: An Ecological Study Data of 185 Countries. Journal of stroke and cerebrovascular diseases. 2020;29(9):105089.

11. Arora A, Jha AK, Alat P, Das SS. Understanding corona phobia. Asian Journal of Psychiatry. 2020.

12. Duan, Li; Zhu, Gang. Psychological interventions for people affected by the COVID-19 epidemic. The Lancet Psychiatry. 2020;7(4):300-2.

13. Islam S, Ferdous Z, Potenza MN. Panic and survey early generalized anxiety during the COVID-19 Pandemic among Bangladeshi people: An online pilot in the outbreak. Journal of affective disorders. 2020;276:30-37.

14. Shi L, Lu ZA, Que JY, Huang XN, Liu L, Ran MM et al. Prevalence of and Risk Factors Associated With Mental Health Symptoms Among the General Population in China, the Coronavirus Disease 2019 Pandemic. X JAMA network. 2020.

15. Wulandari P, Hidayat P. General Anxiety DisorderRelated Coronavirus Disease-19 Outbreak in Indonesia: A Case Report. Open Access Macedonian Journal of Medical Sciences. 2020;8(T1):36-8.

16. Rosenstock S, Jørgensen T, Bonnevie O, Andersen L. Risk factors for peptic ulcer disease: a populationbased prospective cohort study comprising 2416 Danish adults. Gut. 2003;52(2):186-93.

17. Cheng Y, Macera CA, Davis DA, Blair SA. Physical activity and peptic ulcers. Western Journal of medicine, 2000;173(2):101-107.

18. Buckle P, Devereux J. The nature of work-related neck and upper limb musculoskeletal disorders: Applied Ergonomics. 2002;33:207-217. 
19. Brandt LPA, Andersen JH, Lassen CF, Kryger A, Overgaard E, Vilstrup I, et al. Neck and shoulder symptoms and disorders among Danish computer workers. Scand J Work Environ Health. 2004;30:399409.

20. Sauter SL, Schleifer LM, Knutson SJ. Work posture, workstation design, and musculoskeletal discomfort in a VDT data entry task. Hum factors. 1991;33(2):15167.

21. Al-Hashel JY, Ismail II. Impact of coronavirus disease 2019 (COVID19) pandemic on patients with migraine: a web-based survey study. The Journal of Headache and Pain. 2020;21:115.

22. Ilaria Montagni, Elie Guichard, Claire Carpenet, Christophe Tzourio, Tobias Kurth. Screen time exposure and reporting of headaches in young adults: A cross-sectional study International headache society. 2016;11(36).
23. Lim ECH, Seet RCS, Lee KH, Wilder-Smith EPV, Chuah BY, Ong BKC. Headaches, and the N95 facemask amongst healthcare providers, Acta Neurologica Scandinavica. 2006;113(3):199-202.

24. Sinha N, Balayla G. Hydroxychloroquine and COVID-19. Postgraduate medical journal. http://orcid.org/0000-0002-6028-3271. Accessed on $10^{\text {th }}$ January, 2021.

25. Reinhardt I, Euphrosyne, Mayfrank G, Zielasek J. Use of Telepsychiatry in Emergency and Crisis Intervention: Current Evidence, Current Psychiatry Reports. 2019;21:63.

Cite this article as: Yedlapalli M, Kiran SP, Potti S, Devikala A, Kambar C. A study on disease burden and treatment among patients attending teleconsultation during lockdown period. Int J Basic Clin Pharmacol 2021;10:434-41. 